ISSN 1112-9867

\title{
THE EFFECTS OF DIFFERENT VOLUMES OF DYNAMIC STRETCHING ON 20-M REPEATED SPRINT ABILITY PERFORMANCE
}

\author{
A. Ishak ${ }^{*}$, H. Ahmad, N. I. Mohamed and N. A. Rosman \\ Faculty of Sport Science and Coaching, Universiti Pendidikan Sultan Idris, Malaysia
}

Published online: 10 November 2017

\begin{abstract}
The purpose of this within-subjects counterbalanced design study is to elucidate the effects of different volumes of dynamic stretching on Repeated Sprint Ability (RSA) performance. Thirteen male team sport athletes perfromed a repeated sprint ability test consisting of a maximal 6 x 20 meter sprint (with 30s active recovery between each sprint) following different volumes of dynamic stretching (DSS1, DSS2 and DSS3). The results showed no significant difference for all parameters between all the all dynamic stretching volumes. Results show that any of the dynamic stretching volumes may be used as a warm up prior to the repeated sprints session. However, DSS1 confers some advantage in terms of lesser times, though not statistically significant for BST, MST and TST
\end{abstract}

Keywords: fatigue; set; repetition; specific warm-up; recovery.

Author Correspondence, e-mail: asmadi@fsskj.upsi.edu.my

doi: http://dx.doi.org/10.4314/jfas.v9i6s.76

\section{INTRODUCTION}

Dynamic stretching prior to sporting performance has been recognized as an essential component in warming up [1]. It's has been defined as a controlled movement through the active range of motion for each joint $[2,3,4]$. Dynamic stretching consists of exercises that are 
functionally bases and incorporate sport specific movement to prepare the body for activity. Recently, dynamic stretching has recommended as a substitute to other forms of stretching particularly to the static stretching during the warm-up [5,6,7]. Dynamic stretching has been associated with altered in performance changes by $(+1.3 \%)$ compared to other form of stretching techniques (static and PNF) [8]. Meanwhile, dynamic stretching during warm-up showed to positively affect $20 \mathrm{~m}$ sprint performance [9]. A study was recently conducted to examine the effects of 8 weeks of dynamic stretching during warm-up on repeated and single sprint performance [10]. Thirteen seven male soccer players was randomized assigned into 3 groups consists of active dynamic stretching (ADS), static dynamic stretching (SDS) and control group. They performed 6 x 20m RSA in straight-line sprints, interspersed with 20s active recovery in between. The statistical analyses revealed that no significant different were found between groups or time effects for all RSA variables (best time, total time and fatigue index).

However, the inconsistency in the outcomes may be because of difference in the numerous methodologies employed in the various studies such as the type of dynamic stretching performed, the number of stretched muscle groups and the volume of stretching (number or set of drills performed per each muscle groups) [11]. Numerous studies have documented various protocols in the stretching volume used, ranging from a single set to seven sets [4, $12,13,14,15]$. A previous study found that two sets of $20 \mathrm{~m}$ of active dynamic stretching showed a significantly decreased time sprint and the researchers concluded that two sets of active dynamic stretching has preferable benefits on sprint performance [9]. However, in another similar study, the researchers did not find any significant changes in RSA variables after two sets of active dynamic stretching (ADS) [10]. It appears that the effects of the different volumes of dynamic stretching on RSA is not concrete due to the conflicting findings found by the previous researchers. Thus, the number of sets that should be used for dynamic stretching during warm-ups to maintain the time in repeated sprint for team sport has not been determined. Unfortunately, the effects of dynamic stretching in warm-up on the repeated sprint ability in team sport is still lacking and needs more research before any definitive conclusion can be made. Therefore, the influence of various volumes in the dynamic stretching protocols on repeated sprint ability (RSA) needs to investigated to 
elucidate which dynamic stretching volume is beneficial to enhance and maintain sprint times and reduce fatigue.

\section{EXPERIMENTAL METHODS}

Thirteen male team sport athletes (Handball $n=15$, and futsal $n=15$ ) with the mean age of $22.1 \pm 06$ years, height $(166.9 \pm 6.6 \mathrm{~cm})$, body mass $(67.5 \pm 8.0 \mathrm{~kg})$, BMI $\left(24.1 \pm 2.5 \mathrm{~kg} / \mathrm{m}^{2}\right)$ had participated in this within-subjects counterbalanced designed study. All the subjects were students pursuing Sport Science and Physical Education degrees at Universiti Pendidikan Sultan Idris, Malaysia. The subjects also trained regularly for three days a week for at least one year. All subjects had no history of recent muscloskeletal injuries before participating in this study, and none of them were taking any dietary supplements or pharmaceutical drugs that may affect performance during the study. All subjects were free of illness during the study period. Informed written consent form was obtained from all participants for this study which was approved by the Human Ethics Commitee of the Department Sport Science, Universiti Pendidikan Sultan Idris, Malaysia before the commencement of the measurement sessions.

\section{Procedures}

The subjects attended a total of four sessions, including a session for familiarization of experimental protocol. Testing were conducted at the same of the day for each subject (between 07:00 and 11:00 am) to ensure no diurnal variations and under standard enviromental conditions $\left(25^{\circ} \mathrm{C}\right.$ and $70 \%$ relative humidity). Subjects ingested plain water ad-libitum to ensure adequate hydration status. During the familiarization session, subject's data pertaining to age, height, body mass and BMI were collected following which they then completed stretching exercises and 20-m repeated sprint ability (RSA) protocols. For the remaining sessions, subjects completed the sessions over the subsequent nine days, separated by approximately 72 hours between each session.

\section{Warm-up and stretching protocols}

Subjects performed general warm-up with a 10 minute running on the treadmill at $50 \%$ of $\mathrm{VO}_{2 \max }$ with speed set at 5-6 $\mathrm{km} / \mathrm{h}$ until heart rate rose to $110-130 \mathrm{bpm}$. A heart rate monitor 
was fitted to the subject during all session's measurement. After completion of the warm-up, subjects were asked to perform one of the three different volumes of the dynamic stretching in separate measurement sessions.

The dynamic stretch protocols incorporating five active dynamic exercises, designed to mimic parts of sprint cycle and to dynamically stretch the lower body musculature mainly used in sprinting such as gastrocnemius, gluteals, hamstrings, quadriceps and hip extensor. All the dynamic stretching exercises were performed within a distance of 20 meters. Subjects performed 20 repetitions for each exercise with gentle and smooth movements and assisted by two primary research assistants. The participants were instructed to maintain a vertical torso, with knee toward chest to ensure to control the stability postural. A rest period of 30 -s was allowed between sets. The dynamic stretches were base on the stretching protocol previously used [16].

\section{Specific Warm-up}

After completing dynamic stretching, subjects were asked to perform approximately five minutes of incremental intermittent sprints. This activity is in line with previouis suggestion that subjects should be encouraged to end the warm-up with period of activity similar to the activity when they are to perform before beginning any athletic performance [17]. The component of specific warm-up consists of $3 \times 30 \mathrm{~m}$ at striding pace, $3 \times 20 \mathrm{~m}$ at full pace, and $2 \times 30 \mathrm{~m}$ at full pace with walking back to starting point to recovery. The participants were allowed given two minutes of passive recovery in between. The rest period before the actual experimental trials were approximately five minutes.

\section{0-m Repeated Sprint Ability (RSA)}

This present study used a 20 meter repeated sprint protocol. The $20-\mathrm{m}$ RSA performance is considerd to be a relevant performance parameter important to success in all sports involving sprint [18] and 20-m represent the mean sprint distance in field-based team sports [19]. The subjects were instructed to perform a RSA with consists of six repetitions of 20 meters sprint. Every repetition was interspersed with 30 s of active recovery. Strong verbal encouragement were provide to each subject during all RSA trials. Heart rate was measured throughout the 
test by using heart rate monitor and RPE was recorded by used Borg's 6-20 scale. Blood lactate was taken before and five minutes the end of set for RSA trials, a fingertip blood sample $(5 \mu \mathrm{L})$ were collect. A total of time sprint (TST), mean time sprint (MST), best time sprint (BST), RPE and Fatigue Index (FI) were recorded after each repetitions for both RSA protocols.

\section{Statistical Analysis}

All statistical analyses were performed using Statistical Package for Social Sciences (SPSS version 23.0). Descriptive statistics (mean $\pm \mathrm{SD}$ ) and the repeated measure analysis of variance (ANOVA; 3 condition x 1 protocol) was used to determine if significant differences exist between 3 conditions (DS1, DS2, and DS3) and 20-m repeated sprint ability protocols (17). The statistical significant will be set at $p \leq 0.05$.

\section{RESULTS AND DISCUSSION}

Descriptive statistics of the primary varibles are presented in Table 1 . The result of repeated measure ANOVA indicated that no significant different between DSS1, DSS2 and DSS3 on the primary varibles RPE, Lac, TST, MST, BST and FI for 20-m repeated sprint ability.

Table 1. Descriptive statistics of primary varibles (mean $\pm \mathrm{SD}$ )

\begin{tabular}{lcccc}
\hline & DSS1 & DSS2 & DSS3 & P value \\
\hline RPE & $10.46 \pm 2.43$ & $10.23 \pm 1.96$ & $10.76 \pm 1.92$ & 0.62 \\
Lac & $11.07 \pm 3.00$ & $8.96 \pm 2.80$ & $9.18 \pm 2.22$ & 0.36 \\
TST & $19.99 \pm 1.01$ & $20.09 \pm 0.84$ & $20.21 \pm 1.21$ & 0.52 \\
MST & $19.11 \pm 0.81$ & $19.37 \pm 0.73$ & $19.45 \pm 1.10$ & 0.13 \\
BST & $3.18 \pm 0.13$ & $3.22 \pm 0.12$ & $3.24 \pm 0.18$ & 0.13 \\
FI & $4.54 \pm 2.15$ & $3.72 \pm 2.66$ & $3.88 \pm 1.86$ & 0.56 \\
\hline
\end{tabular}

RPE: Rate of perceived exertion; Lac: Lactate; TST: total sprint time; MST: mean sprint time; BST: best sprint time; FI: fatigue index 


\section{DISCUSSION}

The main finding of this present study showed that the results for all the parameters are not significantly different between all the different volumes of dynamic stretching. Although not significantly different, subjects completing DSS2 fared slightly better for Ratings of Perceived Exertion (RPE), blood lactate levels (Lac) and Fatigue Index (FI). Subjects completing DSS1 generally had lesser times for total sprint time (TST), mean sprint time (MST) and best sprint time (BST). Subjects performing DSS3 did not show any advantage over DSS2 in all the parameters measured but fared slightly better than DSS1 for Lac and FI.

This result can be taken to mean that any of the dynamic stretching volumes may be used as a warm up prior to the repeated sprints session. However, DSS1 confers some advantage in terms of lesser times, though not statistically significant for BST, MST and TST (3.18s, $19.11 \mathrm{~s}$, and $19.99 \mathrm{~s}$ respectively) compared to DSS2 (3.22s, 19.37s, and 20.19s respectively) and DSS3 (3.24s, 19.45s, and 20.21s respectively). For less perception of effort (RPE), lower blood lactate values (Lac) and lower sensations of fatigue (FI), subjects completing DSS2 fared slightly better $(10.23,8.96 \mathrm{mmol} / \mathrm{L}$, and 3.72 respectively) compared to DSS1 (10.46, $11.07 \mathrm{mmol} / \mathrm{L}$, and 4.54$)$ and DSS3 (10.76, $9.18 \mathrm{mmol} / \mathrm{L}$, and 3.88 respectively).

Dynamic stretching has been defined as a controlled movement through the active range of motion for each joint [8]. Post-dynamic stretching performance enhancements have been hypothesized to be due to a number of factors such as an increase in skeletal muscle perfusion caused by prior contractions and relaxation of the musculature $[14,20]$.

Enhanced RSA performance after post-dynamic stretching could be attributed by an enhanced musculotendinous unit (MTU) stiffness [8]. The gain in flexibility have been primary attributed by decreased of musculotendinous unit (MTU) stiffness [21]. The key attributes of dynamic stretching include enhanced motor unit excitability and improved kinaesthetic sense, leading to improve proprioception and pre-activation [22].

The seemingly positive effects of DSS1 in BST, MST and TST for the 20 meter repeated sprints might be related to an enhanced intramuscular coordination. It has been suggested that dynamic stretching exercises consisted of movements that partially copy the sprint cycle, which might be help proprioception and improved the lower extremity muscle coordination [8]. Therefore, the DSS1 conditions may has aided in optimizing the synchronization of 
agonist and antagonist muscle activation, hence improving movement speed during 20-meter RSA performance even though it is possible that fatigue induced by the greater effort for better sprint times (higher speed) may have masked these phenomena.

\section{CONCLUSION}

The general objective of this study was to investigate the effect of different volumes of dynamic stretching (DSS1, DSS2 and DSS3) on repeated sprint ability (RSA 20 meters). To achieve this objective, subjects performed one of the three different sets of dynamic stretching, aforementioned and followed by 20 meters repeated sprints in a counterbalance design.

Results show that there was no significant effect of the different volumes of dynamic stretching undertaken by subjects prior to the 20 meter repeated sprints on the parameters studied. However, DSS1 prior to the repeated sprints do offer some promising performance benefits compared to DSS2 and DSS3 and terms of a reduced BST, MST and TST. DSS2 subjects, however had lesser values for MHR, RPE, Lac and FI.

The finding of this study is that during warm-ups, athletes involved in intermittent sports can use any of the dynamic stretching protocols to stretch their lower muscles in sports that require short sprints (20 meters or less) performances within five minutes of the warm up but DSS1 and DSS2 are probably better used for reduced sprint times.

\section{ACKNOWLEDGEMENTS}

This research is funded by University Research grant (GPU) under the project No: 2016-0202-102-01. The authors wished to thanks Research Management and Innovation Centre (RMIC), Universiti Pendidikan Sultan Idris, assistant reseacher and also special thanks to our subjects from Faculty of Sport Science and Coaching for all the supports given during this research.

\section{REFERENCES}

[1] Sim A Y, Dawson B T, Guelfi K J, Wallman K E, and Young W B. Effects of static stretching in warm-up on repeated sprint performance. J Strength Cond Res., 2009, 23(7): 2155-2162. 
[2] Yamaguchi T, and Ishii K. Effects of static stretching for 30 seconds and dynamic stretching on leg extension power. J Strength Cond Res., 2005, 19: 677-683.

[3] Chaouachi A, Chamari K, Wong P, Castagna C, Chaouachi M, Moussa-Chamari I, and Behm D. G. Stretch and sprint training reduces stretch-induced sprint performance deficits in 13- to 15-year-old youth. Eur J Appl Physiol., 2008, 104(3):515-522.

[4] Jaggers J R, Swank A M, Frost K L, and Lee C D. The acute effects of dynamic and ballistic stretching on vertical jump height, force and power. J Strength Cond Res., 2008, 22: $1844-1849$.

[5] Fletcher I M, Mand Anness R. The acute effects of combined static and dynamic stretch protocols on fifty-meter sprint performance in track-and-field athletes. J Strength Cond Res., 2007, 21:784-787.

[6] Moran K A, McGrath T, Marshall B M, and Wallace E S. Dynamic stretching and golf swing performance. Int J Sport Med., 2009, 30: 113-118.

[7] Pearce A J, Dawson J, Kidgell D J, Zois J, and Carlson J S. Effects of secondary warm up following stretching. Eur J Appl Physiol., 2009, 105: 175-183, 2009.

[8] Fletcher I M, Jones B. The effects of different warm up stretch protocol on 20 meter sprint performance in trained rugby union players. J Strength Cond Res., 2004, 18:784-787.

[9] Turki O, Chaouachi A, Behm D G, Chtara H, Chtara M, Bishop D, Chamari K, and Amri M. The effects of warm-ups incorporating different volumes of dynamic stretching on 10 and $20 \mathrm{~m}$ sprint performance in higly trained male athletes. J Strength Cond Res., 2012, 26(1): $63-72$.

[10] Turki-Belkhiria L, Chaouachi A, Turki O, Chtourou H, Chtar M, Chamari K, Amri M, and Behm D G. Eight weeks of dynamic stretching during warm-ups improves jump power but not repeated or single sprint performance. Eur J Sport Sci., 2014, 14(1) :19-27.

[11] Faigenbaum A D, Kang J, Mcfarland J, Bloom J M, Magnatta J, Ratamess N A, and Hoffman J. Acute effects of different warm-up protocols on anaerobic performance in teenage athletes. Pediatr Exerc Sci., 2006, 17: 64-75.

[12] Papadopoulus G, Siatras T, Kellis S. The effects of static and dynamic stretching exercises on the maximal isokinetics strenght og the knee extensor and flexors. Isokinet Exerc Sci., 2005, 13:285-291. 
[13] Beedle B, Rytter S J, Healy R C, and Ward T R. Pretesting static and dynamic stretching does not affect maximal strength. J Strength Cond Res., 2008, 22:1838-1843

[14] Manoel M E, Michael O, Harris-love M O, Danoff J V, and Miller T A. Acute effects of static, dynamic and proprioceptive neuromuscular facilitation stretching on muscle power in women. J Strength Cond Res., 2008, 22: 1528-1534.

[15] Samuel M N, Holcomb W B, Guadagnoli M. A, Rubley M D, and Wallmann H. Acute effects of static and ballistic stretching on measures of strength and power. J Strength Cond Res., 2008, 22: 1428-1428.

[16] Pearce A J, Dawson J, Kidgell D J, Zois J, and Carlson J S. Effects of secondary warm up following stretching. Eur J Appl Physiol., 2009, 105: 175-183, 2009.

[17] Fradkin A J, Zarzryn T R, Smoliga J M. Effects of warm-up of warming-up on physical performance: A systematic review with meta-analysis. J Strength Cond Res., 2010, 24: $140-148$.

[18] Fletcher I M. The effect of different dynamic stretch velocities on jump performance. Eur J Appl Physiol, 2010, 109, 491-498.

[19] Spencer M, Lawrence S, Rechichi C, Bishop D, Dawson B, and Goodman C. Time-motion analysis of elite field hockey, with special reference to repeated-sprint activity. $\mathrm{J}$ Sports Sci., 2004, 22: 843-850.

[20] Little T, Williams A G. Effects of differential stretching protocols during warm ups on high-speed motor capacities in profesional soccer players. J Strength Cond Res., 2006, 20: 203-207.

[21] Wilson G J, Murphy A J, Pryor J F. Musculotendinous stiffness : its relationship to eccentic, isometric and concentric performance. J Appl Physiol, 1994, 76: 2714-2719.

[22] Mann D, Jones M. Guidelines to the implementation of a dynamic stretching program. J Strength Cond Res., 1999, 21: 53-55.

\section{How to cite this article:}

Ishak A, Ahmad H, Mohamed N I, and Rosman N A. The effects of different volumes of dynamic stretching on 20-m repeated sprint ability performance. J. Fundam. Appl. Sci., 2017, 9(6S), 1041-1049. 